\title{
Biomedical fluid mechanics and fluid-structure interaction
}

\author{
Yuri Bazilevs · Kenji Takizawa · Tayfun E. Tezduyar
}

Received: 23 June 2014 / Accepted: 28 June 2014 / Published online: 15 July 2014

(C) Springer-Verlag Berlin Heidelberg 2014

In 2013, we celebrated the 70th Birthday of Thomas J.R. Hughes, a leader in computational mechanics. With this special issue, we recognize Tom Hughes's contributions and impact in biomedical fluid mechanics and fluid-structure interaction (FSI).

Tom Hughes is a pioneer in computational biomechanics, going all the way back to his PhD work on arterial pulse propagation in 1973, with his landmark work with Charles Taylor and Chris Zarins on patient-specific arterial fluid mechanics modeling in 1996, and with the more recent innovations he brought to computational biomechanics with isogeometric analysis. Inspired by Tom Hughes's work and ideas in computational biomechanics, his students, associates and friends have also been making significant contributions in this area of computational mechanics and advancing the state-of-the-art. We are proud to have a contribution also from Tom Hughes in this special issue.

The special issue a collection of state-of-the-art papers on biomedical fluid mechanics and FSI and closely related topics, with much emphasis on patient-specific modeling. Significant emphasis is placed also on modeling of biomedical devices, such as ventricular assist devices (VADs) and artificial heart valves. While most of the articles focus on the spatial scales at the organ level (e.g., scales of aortic valves and large blood vessels), computational FSI techniques for

Y. Bazilevs

University of California, San Diego, CA, USA

K. Takizawa

Waseda University, Tokyo, Japan

T. E. Tezduyar $(\varangle)$

Rice University, Houston, TX, USA

e-mail: tezduyar@tafsm.org red blood cells, which are at much smaller scales, are also covered.

A number of novel and promising computational methods addressing the key contemporary challenges in cardiovascular fluid mechanics and FSI are presented. (A) Methods that can deal with topological changes in the fluid mechanics domain in FSI computation of heart valves. (B) Accurate and efficient techniques for residence-time calculation in FSI computation of pulsatile VADs. (C) Methods for coupling 3D and 1D cardiovascular simulations and further improving the efficiency through the use of reduced-order modeling. (D) Methods for estimation of zero-stress state in arterial FSI and their use in coronary arterial dynamics computations with medical-image-based anatomical models. (E) Validated computational methods for deployment as part of high-intensity focused ultrasound (HIFU) therapy. (F) Gradient-smoothed CFD (GSM-CFD) methods for high-accuracy hemodynamics computations.

The applications presented in the special issue are of high clinical relevance and are just as impressive as the methods presented. (A) Hemodynamics of intracranial aneurysms. (B) Hemodynamics of the Thoracic Endovascular Repair (TEVAR). (C) FSI of the thoracic aorta and flow analysis based on its geometric characteristics. (D) Hemodynamics and FSI of aortic valves. (E) Shape optimization of pulsatile VADs using FSI to minimize thrombotic risk.

We thank the authors for the effort in preparing their contributions and for meeting the special-issue deadlines. These contributions will undoubtedly help advance computational biomedical fluid mechanics and FSI. 